\title{
Analytical Framework of Forced Convective Two- dimensional MHD Flow of Jeffrey Nano Liquid With Impact of Thermal Radiation and Activation Energy
}

Haroon Ur Rasheed ( $\square$ haroon.csit@suit.edu.pk)

Abdul Wali Khan University

Saeed Islam

Abdul Wali Khan University

Zeeshan Khan

Sarhad University of Science and Information Technology

Waris Khan

Hazara University Mansehra

\section{Research Article}

Keywords: Jeffrey nanofluid, MHD, HAM solution, mixed convection, thermal radiation

Posted Date: July 7th, 2021

DOl: https://doi.org/10.21203/rs.3.rs-672606/v1

License: (c) (i) This work is licensed under a Creative Commons Attribution 4.0 International License.

Read Full License 


\title{
Analytical framework of forced convective two-dimensional MHD flow of Jeffrey nano liquid with impact of thermal radiation and activation energy
}

\author{
Haroon Ur Rasheed*1, Saeed Islam ${ }^{1}$, Zeeshan Khan ${ }^{2}$, Waris Khan ${ }^{3}$ \\ 1Department of Mathematics, Abdul Wali Khan University, Mardan 23200, KP, Pakistan \\ 2Sarhad University of Science and Information Technology Peshawar 25000, KP, Pakistan \\ 3Department of Mathematics and Statistics, Hazara University Mansehra, 21120 KP, Pakistan \\ *Corresponding author email: haroon.csit@suit.edu.pk
}

Abstract: The present communication owns to address the mathematical framework of twodimensional electrically conductive and thermally radiative Jeffrey nanofluid flow by a curved surface. The interaction of a periodic magnetic field with the suspended nanoparticles and mixed convection are critically important due to its application in a broad spectrum. Buongiorno's model, incorporates the effect of thermophoretic force and Brownian movement, describes the nature of Jeffrey nanofluid. The influence of activation energy, viscous dissipation, and thermal radiation effects are reserved. The dimensionless system of differential equations has been diminished from the modeled equations via transformation framework which is solved analytically versus homotopic algorithm. The stability and convergence analysis has been carried out to optimize system parameters and accuracy of the system. The effect of physical constraints on flow field, energy, and concentration of nanoparticles are portrayed via plotted graphs and debated.

Key words: Jeffrey nanofluid, MHD, HAM solution, mixed convection, thermal radiation.

\section{Introduction}

The advancements in nanofluid technology increased largely during the past few years due to their higher thermal performances. Nanofluid is a suspension of small size $(1-100 \mathrm{~nm})$ particles into a base liquid. Normally such fluids are made by using a mixture of solid particles into base fluids like water, engine oil, ethylene glycol (EG) and many other base liquids. The structure of nanoparticles consists of metal carbide, nitride, metal oxide, and even nanoscale liquid droplets [1]. Such particles have shape of sphere, tubular, rod-like etc. Choi's investigation [2] shows that the implementation of the nanofluids is utilized in vast range of industrial manufacturing processes like textile, transportation, paper production, electronic equipment, energy production and many others. The basic characteristics of nanofluids are their thermal conductivities which are much higher than the other base fluids. The combination of such substances offers us a medium for heat transfer that behaves as a fluid. In fact, addition of nano size particles in the base fluids develops thermal behavior due to which heat transfer characteristics are increased substantially. Further, the magnetohydrodynamic (MHD) nanofluids are significant in engineering and industrial processes. Such fluids are used in optical modulators, optical gratings, optical switches, tunable optical fiber filters, stretching of plastic sheets and metallurgy, polymer industry, and other applications. Many metallurgic procedures involve drawing continuous strips/filaments through a nanofluid to cool them. Such strips are sometimes stretched processes the drawing and thinning of copper processes. In such situations, the quality and wanted features of the final product is obtained by drawing such strips into electrically conducting fluid. Magnetic nanoparticles have a key role in the construction of loudspeakers, magnetic cell separation, hyperthermia, drug delivery etc. The general applications of nanofluids include cooling of vehicle, making new types of fuel, saving of fuel in electric power generation units, cancer therapy, imaging and sensing and other useful 
applications. References [3-10] show some recent attempts on this topic. Ahmad et al. [11] investigated MHD flow of three-dimensional unsteady Jeffrey nanofluid with thermal radiation and Joule heating in view of porous medium. Fiza et al. [12] studied homotopic solution 3D steady state Jeffrey liquid in rotating channel subject constant magnetic field with the application of Hall current. Ahmad et al. [13] proposed analytical solution of unsteady 3D flow of Jeffrey nanofluid past a bi-directional oscillatory surface. The influence of Brownian movement and thermophoretic force are analyzed by incorporating Buongiorno's model. Rasheed et al. [14] discussed the consequences of unsteady hydromagnetic thermally radiative Jeffrey nanofluid flow over a vertical surface with viscous dissipation and Joule heating. Naidu et al. [15] debated numerical implication of partial slip effect on MHD Jeffrey nanofluid flow including microorganisms over a stretching surface. Ge-JiLe et al. [16] examine numerically the implication of slip flow of Jeffrey nanofluid by a stretching surface with entropy generation and activation energy. Noor et al. [17] studied heat mass transfer effect on thermally radiative and chemically reactive time dependent Jeffrey nanofluid flow in a permeable channel with slip condition and heat reservoir source. Shahzada et al. [18] scrutinized numerical solution of steady state 2-dimensional Jeffrey nanofluid by an elongated surface. Hayat et al. [19] studied entropy generation in flow with $\mathrm{Ag}$ and $\mathrm{Cu}$ nanoparticles over a rotating circular disk with variable thickness and thermal radiation. Krishnamurthy et al. [20] investigated numerical solution of 2-dimensional slip flow by a curved surface in view of porous matrix. Kumar et al. [21] examined thermally radiative Marangoni flow of Casson nanofluid in view of extended surface with chemical reaction, heat reservoir source and heat generation mechanism. Roja et al. [22] debated numerical solution of thermally radiative hydromagnetic flow of micropolar nanofluid by an inclined curved surface with entropy generation, Joule heating and multiple slip effects. Hamid et al. [23] examined convection and heat transfer features in MHD flow of water based CNTs in a fin-shaped cavity with thermal radiation and viscous dissipation effect. Khan et al. [24] developed numerical algorithm to analyzed EyringPowell nanofluid by nonlinear curved surface by incorporating Brownian thermophoretic effects in the model equations. Khan et al. [25] explored various mechanism to developed heat and mass transfer rates by considering different permeable cavities for innumerable fluid models. Some recent and interesting attempt on this topic are provided in [26-31] for innumerable fluid models to understand heat transfer mechanism.

\section{Mathematical model of the problem}

Here, we assumed magnetohydrodynamics flow of Jeffrey nanofluid in view of vertically extending sheet enclosure by a uniform magnetic field and thermal radiation. The fluid flow is expected to be incompressible and laminar. The applied magnetic field is taken as $B_{x}=0, B_{y}=B_{0} \sin \left(\frac{\pi x}{\lambda}\right)$ with strength $B_{0}$. The Reynolds number is small enough such that the influence of induced field is weaker in comparison to the applied magnetic field. Therefore, induced magnetic effect is zero. The electric field is absent and physical properties are constant and independent of temperature. Fig. 1 particularizes the physical configuration of modeled flow problem. 


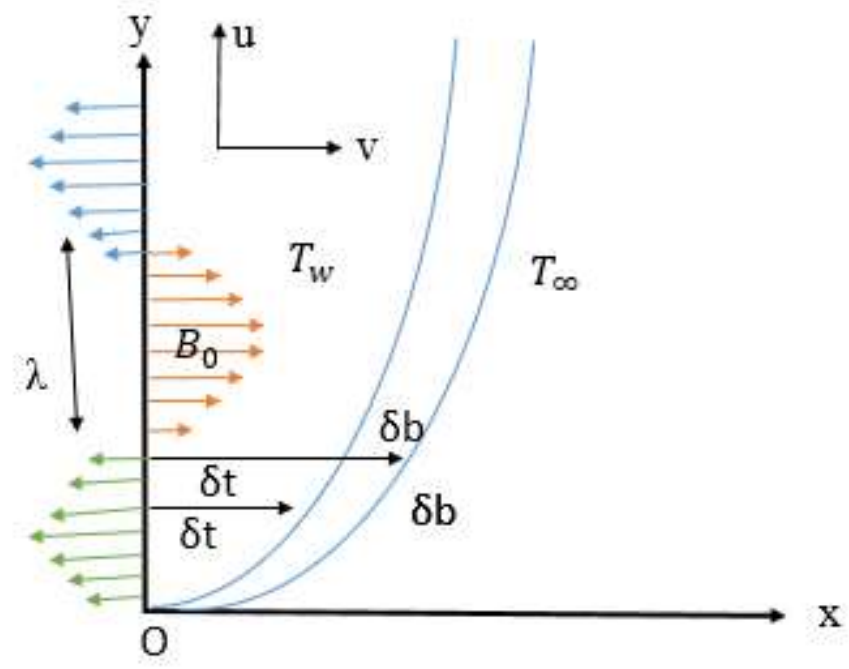

Fig.1 Physical sketched and flow patent of the problem

where $G r$ Grashof number, $\lambda$ wave length, $\tau$ dimensionless time, $T$ dimensionless time, $C$ is the dimensionless concentration profile, $U, V$ are dimensionless velocities, $u^{*}, v^{*}$ are the dimensional velocities and $\tau^{*}$ is the dimensional time. Thus, the fundamental flow equations turn into the following form:

$$
\begin{aligned}
& \frac{\partial u^{*}}{\partial x^{*}}+\frac{\partial v^{*}}{\partial y^{*}}=0 \\
& \frac{\partial u^{*}}{\partial t^{*}}+u^{*} \frac{\partial u^{*}}{\partial x^{*}}+v^{*} \frac{\partial u^{*}}{\partial y^{*}}=g \beta\left(T^{*}-T_{\infty}^{*}\right)+g \beta^{*}\left(C^{*}-C_{\infty}^{*}\right)-\frac{B_{0}^{2} \sigma}{\rho} \sin ^{2}\left(\pi x^{*}\right) u^{*}+ \\
& \frac{v}{1+\lambda_{2}}\left[\frac{\partial^{2} u^{*}}{\partial y^{* 2}}+\lambda_{1}\left(\frac{\partial^{3} u^{*}}{\partial t^{*} \partial y^{* 2}}+u^{*} \frac{\partial^{3} u^{*}}{\partial x^{*} \partial y^{* 2}}+v^{*} \frac{\partial^{3} u^{*}}{\partial y^{* 3}}-\frac{\partial u^{*}}{\partial x^{*}} \frac{\partial^{2} u^{*}}{\partial y^{* 2}}+\frac{\partial u^{*}}{\partial y^{*}} \frac{\partial^{2} u^{*}}{\partial y^{*} \partial x^{*}}\right)\right] \\
& \frac{\partial T^{*}}{\partial t^{*}}+u^{*} \frac{\partial T^{*}}{\partial x^{*}}+v^{*} \frac{\partial T^{*}}{\partial y^{*}}=\frac{\kappa}{\rho c_{p}} \frac{\partial^{2} T^{*}}{\partial y^{* 2}}-\frac{1}{\rho c_{p}} \frac{\partial q_{r}}{\partial y^{*}}+ \\
& \frac{v}{c_{p}\left(1+\lambda_{2}\right)}\left[\left(\frac{\partial u^{*}}{\partial y^{*}}\right)^{2}+\lambda_{1}\left(u^{*} \frac{\partial u^{*}}{\partial y^{*}} \frac{\partial^{2} u^{*}}{\partial x^{*} \partial y^{*}}+v^{*} \frac{\partial u^{*}}{\partial y^{*}} \frac{\partial^{2} u^{*}}{\partial y^{* 2}}\right)\right]+\tau\left[D_{B}\left(\frac{\partial T^{*}}{\partial y^{*}} \frac{\partial C^{*}}{\partial y^{*}}\right)+\frac{D_{T}}{T_{\infty}^{*}}\left(\frac{\partial T^{*}}{\partial y^{*}}\right)^{2}\right] \\
& \frac{\partial C^{*}}{\partial t^{*}}+u^{*} \frac{\partial C^{*}}{\partial x^{*}}+v^{*} \frac{\partial C^{*}}{\partial y^{*}}=D_{B}\left(\frac{\partial^{2} C^{*}}{\partial y^{* 2}}\right)+\frac{D_{T}}{T_{\infty}^{*}}\left(\frac{\partial^{2} T^{*}}{\partial y^{* 2}}\right)
\end{aligned}
$$

With boundary postulates 
at $y^{*}=0, u^{*}=\frac{v}{\lambda}(G r)^{\frac{1}{2}}, v^{*}=0, T^{*}=T_{w}^{*}, C^{*}=C_{w}^{*}$ and

at $y^{*} \rightarrow \infty, u^{*} \rightarrow 0, \frac{\partial u^{*}}{\partial y^{*}} \rightarrow 0, T^{*} \rightarrow T_{w}^{*}, C^{*} \rightarrow C_{w}^{*}$

whereas, $\beta$ denote thermal expansion coefficient, $\beta^{*}$ concentration expansion, $T_{w}^{*}$ wall temperature, $C_{w}^{*}$ species concentration at wall, $b$ stretching constant, $v$ kinetic viscosity, $\rho$ denote fluid density, $\lambda_{1}$ relaxation time, $\lambda_{2}$ denote ratio of relaxation to retardation time, $\kappa$ represent thermal conductivity, $c_{p}$ specific heat, $q_{r}$ is the unidirectional heat flux, $D_{B}$ represent Brownian diffusion coefficient, $D_{T}$ thermophoresis diffusion coefficient. The heat flux term by incorporating the Roseland approximation is defined by the relation as $q_{r}=-\frac{4 \sigma_{S}}{3 k_{e}} \frac{\partial T^{* 4}}{\partial y^{*}}$

By incorporating following transformation to nondimensional quantities:

$$
\begin{aligned}
& Y=\frac{y^{*}}{\lambda} G r^{\frac{1}{4}}, X=\frac{x^{*}}{\lambda}, u^{*}=\frac{v}{\lambda} G r^{\frac{1}{2}} U, v^{*}=\frac{v}{\lambda} G r^{\frac{1}{4}} V, \tau^{*}=\frac{v}{\lambda^{2}} G r^{\frac{1}{2}} t^{*}, T^{*}=T\left(T_{w}^{*}-T_{\infty}^{*}\right)+T_{\infty}^{*}, \\
& C^{*}=C\left(C_{w}^{*}-C_{\infty}^{*}\right)+C_{\infty}^{*}
\end{aligned}
$$

In view of Eq. (6) the non-dimensional Eq. (1) is justified routinely and Eqs. (2)- (5) reduced into the dimensionless state:

$$
\begin{aligned}
& \frac{\partial U}{\partial X}+\frac{\partial V}{\partial Y}=0 \\
& \frac{\partial U}{\partial \tau}+U \frac{\partial U}{\partial X}+V \frac{\partial U}{\partial Y}=(G r) T+(G m) C-M^{2} \sin ^{2}(\pi X) U+ \\
& \frac{1}{1+\lambda_{2}}\left[\frac{\partial^{2} U}{\partial Y^{2}}+K\left(\frac{\partial^{3} U}{\partial \tau \partial Y^{2}}+U \frac{\partial^{3} U}{\partial X \partial Y^{2}}+V \frac{\partial^{3} U}{\partial Y^{3}}-\frac{\partial U}{\partial X} \frac{\partial^{2} U}{\partial Y^{2}}+\frac{\partial U}{\partial Y} \frac{\partial^{2} U}{\partial Y \partial X}\right)\right] \\
& \frac{\partial T}{\partial \tau}+U \frac{\partial T}{\partial X}+V \frac{\partial T}{\partial Y}=\frac{E c}{\left(1+\lambda_{2}\right)}\left[\left(\frac{\partial U}{\partial Y}\right)^{2}+\beta\left(U \frac{\partial U}{\partial Y} \frac{\partial^{2} U}{\partial X \partial Y}+V \frac{\partial U}{\partial Y} \frac{\partial^{2} U}{\partial Y^{2}}\right)\right] \\
& +N b \frac{\partial T}{\partial Y} \frac{\partial C}{\partial Y}+N t\left(\frac{\partial T}{\partial Y}\right)^{2}+\frac{1}{\operatorname{Pr}}\left(1+\frac{4 R}{3}\right) \frac{\partial^{2} T}{\partial Y^{2}} \\
& \frac{\partial C}{\partial \tau}+U \frac{\partial C}{\partial X}+V \frac{\partial C}{\partial Y}=\frac{1}{L e}\left(\frac{\partial^{2} C}{\Delta Y^{2}}+\left(\frac{N t}{N b}\right) \frac{\partial^{2} T}{\Delta Y^{2}}\right)
\end{aligned}
$$

Following are boundary criterions in dimensionless variables:

at $Y=0: U=1, V=0, T=1, C=1$ and

at $Y \rightarrow \infty: U \rightarrow 0, V \rightarrow 0, T \rightarrow 0, C \rightarrow 0$ 
The variables appearing in Eqs. (8)- (10) are signified as:

$$
\begin{aligned}
& M=\frac{\sigma B_{0}^{2} \lambda^{3}}{\rho v^{2}}, G m=\frac{g_{x} \beta_{T}\left(C_{w}^{*}-C_{\infty}^{*}\right) \lambda^{3}}{v^{2}}, R=\frac{4 \sigma_{0} T_{\infty}^{3} \lambda^{3}}{\kappa\left(a+\sigma_{s}\right)}, \operatorname{Pr}=\frac{v}{\alpha}, L e=\frac{v}{D}, \\
& \Delta=T_{w}-1, N b=\frac{\tau^{*} D_{B}\left(C_{w}^{*}-C_{\infty}^{*}\right)}{v}, N t=\frac{\tau^{*} D_{T}\left(T_{w}^{*}-T_{\infty}^{*}\right)}{v T_{\infty}^{*}}, G r=\frac{g_{x} \beta_{T}\left(T_{w}^{*}-T_{\infty}^{*}\right) \lambda^{3}}{v^{2}}
\end{aligned}
$$

Labeled as magnetic parameter, mass Grashof number, the radiation term, Prandtl number, Lewis number, Brownian factor, thermophoresis constraint, Grashof number and respectively.

The parameters having engineering applications in the present problem are skin-friction and Nusselt numbers elucidated as:

$$
C f=\left.\left(\frac{1+\beta}{1+\lambda}\right) \operatorname{Re}_{x}^{-0.5}\left(\frac{\partial u^{*}}{\partial y^{*}}\right)\right|_{y^{*}=0} \text { and } N u=\left.\operatorname{Re}_{x}^{-0.5}\left(-\frac{\partial T^{*}}{\partial y^{*}}\right)\right|_{y^{*}=0}
$$

\section{Convergence analysis of homotopic solution}

In section, we opted a well-known explicit analytical technique HAM for nonlinear computation. The computational analysis is remained insufficient unless the stability and convergence of the analytical solution are discussed. The derived series solution is mainly depending on the appropriate optimal selection of auxiliary constraints [12-14]. The precise choice of these parameters has a substantial importance in controlling and regulating convergence criteria. In this regard, we plotted and outlined h-curves for nonlinear governing Eqs. (8)- (10) in Fig. 2. The plat part of these curves which is parallel to $x$-axis fixes the allowable region of the convergence. The ranges of acceptable values of the auxiliary constraints for fruitful convergence have been disclosed in Table1. It has been perceived that average squared residual error dwindled via larger order of approximations. 


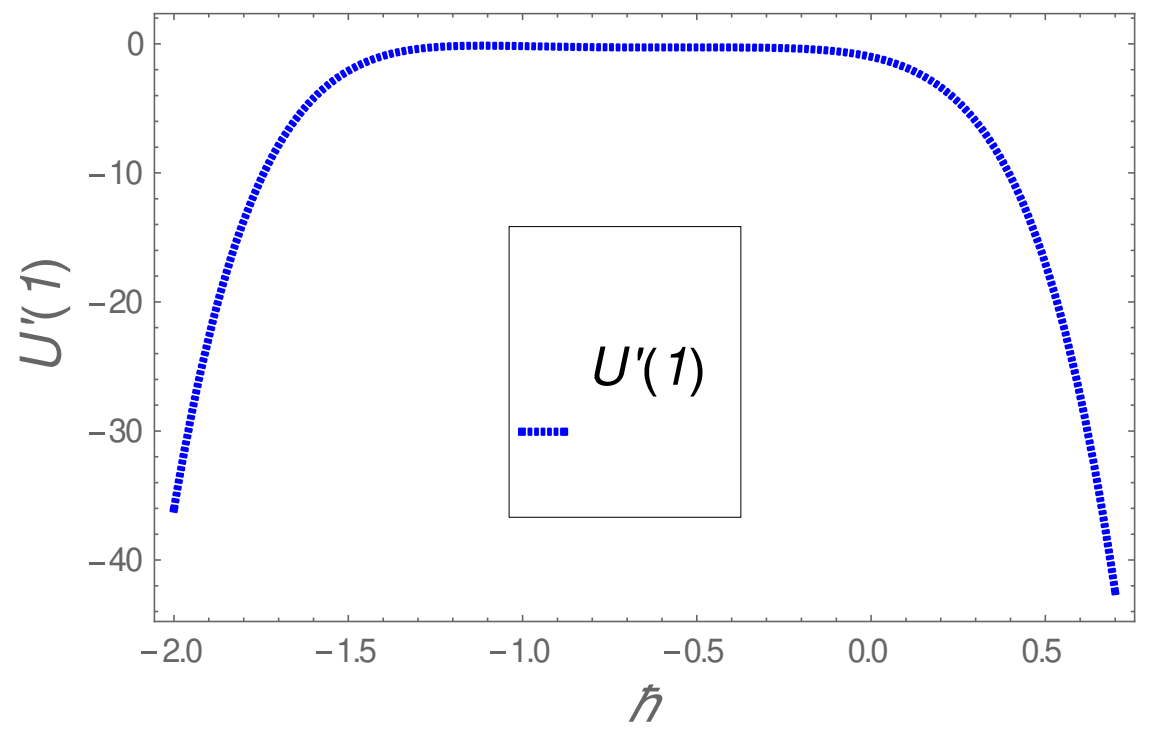

Fig. 2(a). h-curve for velocity

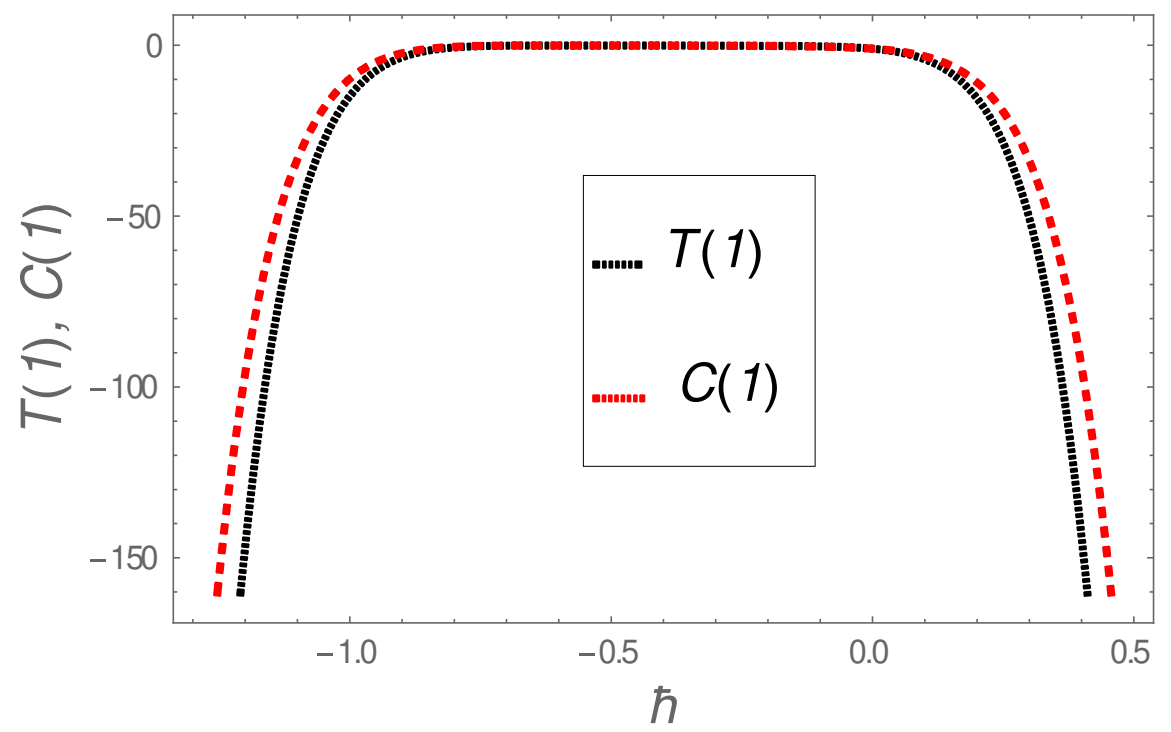

Fig. 2(b). Combined $\mathrm{h}$-curves for temperature and concentration. 
Table 1. The allowable ranges for convergence solutions.

\section{Solutions}

$U(X, Y)$

$T(X, Y)$

$C(X, Y)$
Auxiliary parameters

$\mathrm{h}_{U}$

$\mathrm{h}_{T}$

$\mathrm{h}_{C}$
Convergent intervals

$-1.5 \leq \mathrm{h}_{U} \leq 0.0$

$-0.9 \leq \mathrm{h}_{T} \leq 0.1$

$0.9 \leq \mathrm{h}_{C} \leq 0.2$

\section{Results and discussion}

The discussion segment is devoted to understanding the elementary features of the physical constraints graphically. The nonlinear constitutive flow laws in Eqs. (8) - (10) subject to boundary postulates, in Eq. (11), are tackled via homotopic technique. The consequences of relevant parameters on velocity, energy, and concentration field are explored through plotted graphs in Figures $3-20$.

By

letting

$M=0.4, \operatorname{Pr}=1.0, N t=N b=0.5, \beta=0.2, G r=0.3, G m=0.2, L e=1, R=0.2$, and $E c=0.1$

for

computational analysis.

The result of radiation constraint $(R)$ on fluid velocity is described in Fig 3. From this graph it can be perceived that velocity field upsurges with the intensifying values of radiation constraint. Fig. 4 explains the velocity fields $(U)$ for different values of magnetic $(M)$ parameter. It has been perceived that higher magnetic field diminished nanofluid flow. In reality when magnetic field is applied to fluid it intensifying viscosity of the fluid. In consequence, fluid particles experience resistance to flow due to which velocity retards. It is of great engineering interest that the yield stress of fluid flow can be measured correctly through a variation in magnetic strength. Figure 5 depicts variations in thermal field $(T)$ subjected to larger radiation parameter $(R)$. This figure discloses temperature development for higher thermal radiation factor. In fact, the working fluid gets additional heat energy subject to radiation parameter. In consequence, temperature upsurges. Variations in thermal field curves for diverse values of the magnetic constraint $(M)$ is established in Fig. 6. It has been detected from this graph that the thermal energy curves augmented for intensifying values of magnetic parameter. Furthermore, an increment in $(M)$ leads to decline elastic stress variable. Consequently, fluid temperature augmented in the neighborhood of the flow configuration. Moreover, due to the Joule heating and thermal dissipation effects, extra heat energy flow to working fluid, which, developed thermal energy in the boundary layer region. Attributes of $(E c)$ on temperature distribution $(T)$ curves perceived in Fig. 7. It is seen clearly from this plot that the temperature upsurges for the escalating values of $(E c)$ constraint effectively. This augmentation in the fluid temperature is largely because of the fact that the frictional viscous heating in nanofluid reasons to allow heat energy into the working nanofluid due to which the temperature augments in the boundary layer section. Similarly, the presence of viscous dissipation effects in the energy equation, the temperature profile is boosted. When 
Eckert number is zero it signifies absence of viscous dissipation effects. Fig. 8 unveils the influence of $(\mathrm{Nb})$ on temperature field. The arbitrary motion of nanoparticles boosts for larger $(\mathrm{Nb})$ value developed the kinetic energy of the fluid particles due to which additional thermal energy is produced. Hence, fluid temperature upsurges. Fig. 9 predicts variations in thermal field curves boosts through larger thermophoresis constraint $(N t)$. Here, one can perceive that $(T)$ is increasing function of thermophoretic force. Physically, thermophoretic force upsurges when $(N t)$ is augmented. Such force assists to escaped nanoparticles by hotter towards colder part. In consequence, fluid temperature boosts. The higher values of $(\mathrm{Pr})$ diminished conduction and develop heat transfer rate. Physically, larger Prandtl number significantly reduced thickness of the thermal boundary layer. Consequently, thermal field curves dwindle shown in Fig. 10. Figs. 11 and 12 reveals the impact radiation and magnetic parameters on concentration profile. Here, one can perceived that heat mass transference coefficient boosts through higher radiation constraint consequently, $C$ escalates and diminishes with increment of magnetic parameter. Attributes of $(N t)$ parameter on concentration field is unveiled in Fig. 13. Clearly $(C)$ boosts via larger $(N t)$ . Physically higher thermophoresis constraint yields an upsurge in thermophoretic parameter which cause the frequently movement of the nanoparticles from region of higher to lower temperature. Thus, concentration field escalates. The contribution of $(\mathrm{Nb})$ on concentration profile is exemplified in Fig. 14. It is found that larger data of Brownian parameter, fluid concentration diminishes. Fig. 15 portrays variation in concentration profile for innumerable values of $(L e)$. Further, $(L e)$ defines the ratio of thermal diffusivity to mass diffusivity and it is used to describe the fluid flow where there is simultaneous heat mass transfer via convection. Thus, the concentration diminishes subject to larger Lewis number. The result of magnetic parameter on $C f$ and $N u$ is captured in Figs. 16 and 17 are diminishing considerably keeping to the retarding effects of $(M)$ on flow field. Figure 18 outlines the features of Nusselt number for the higher values of radiation parameter. Clearly Nusselt number rises when $(R)$ is increased which created sinusoidal pattern. On can perceived that higher magnetic parameter augmented kinetic energy between fluid particles. In consequence fluid temperature increases. The streamlines are presented against different values of magnetic parameter in Figs. 19 and 20. Physically it occurs because of a resistive Lorentz force of a magnetic parameter. 


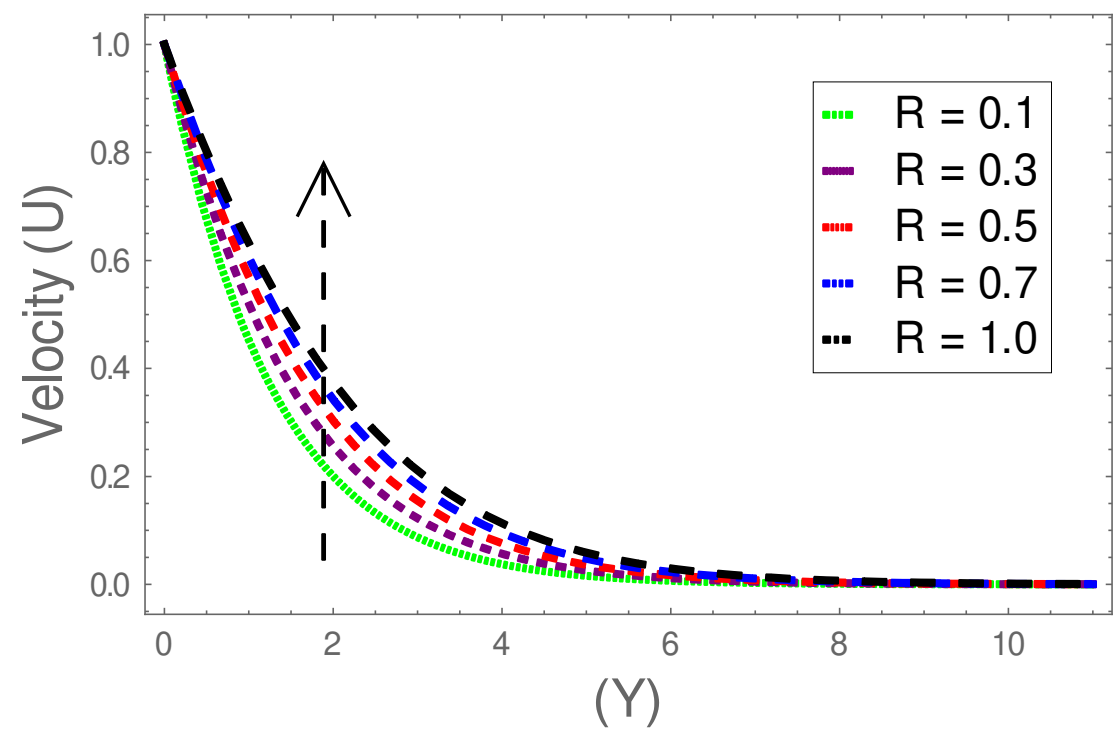

Fig. 3. Variation of $(R)$ on velocity

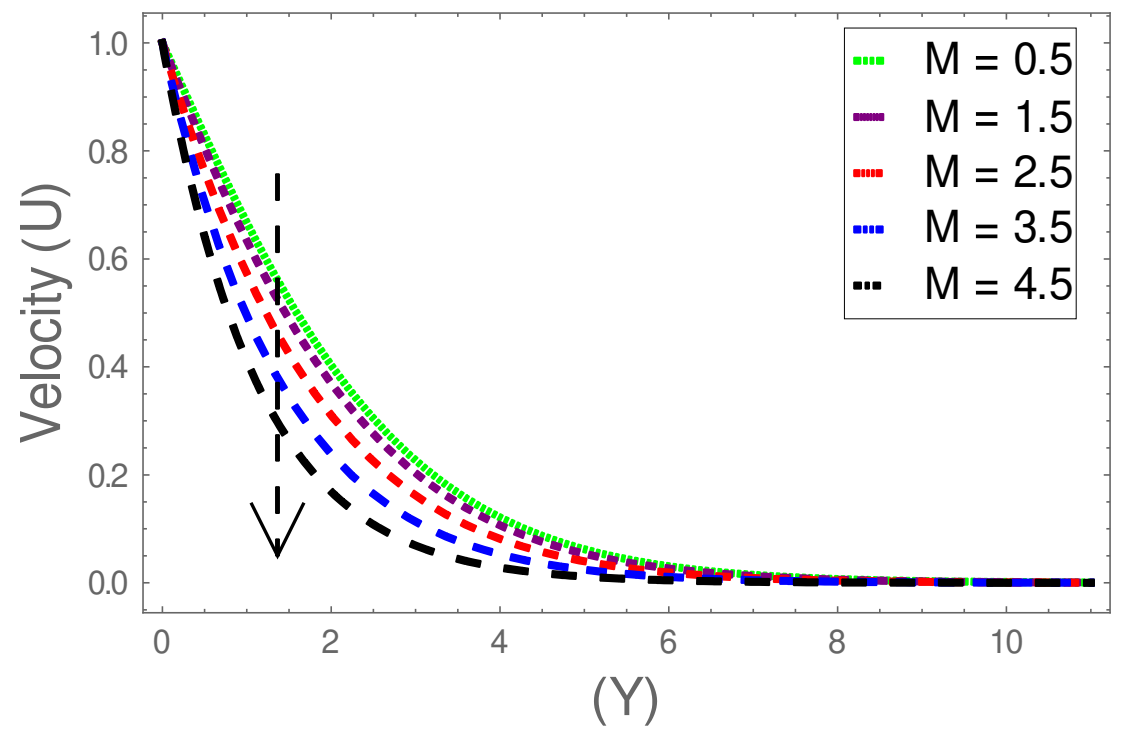

Fig. 4. Variation of $(M)$ on velocity 


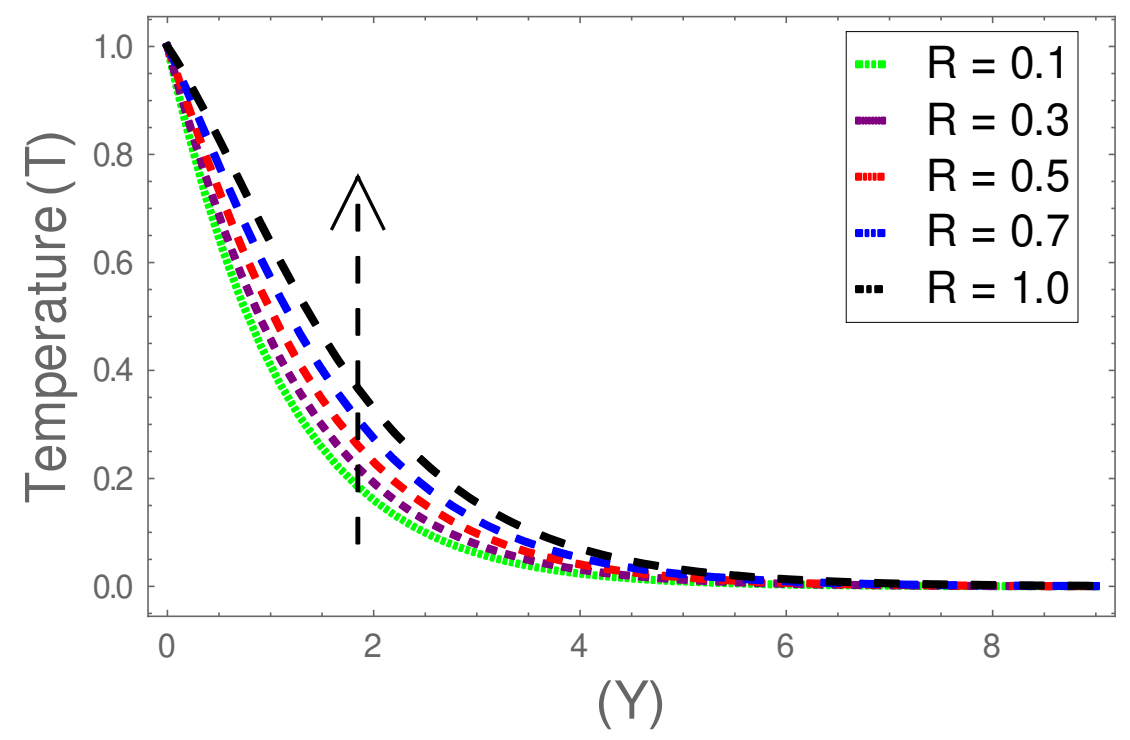

Fig. 5. Variation of $(R)$ on thermal field

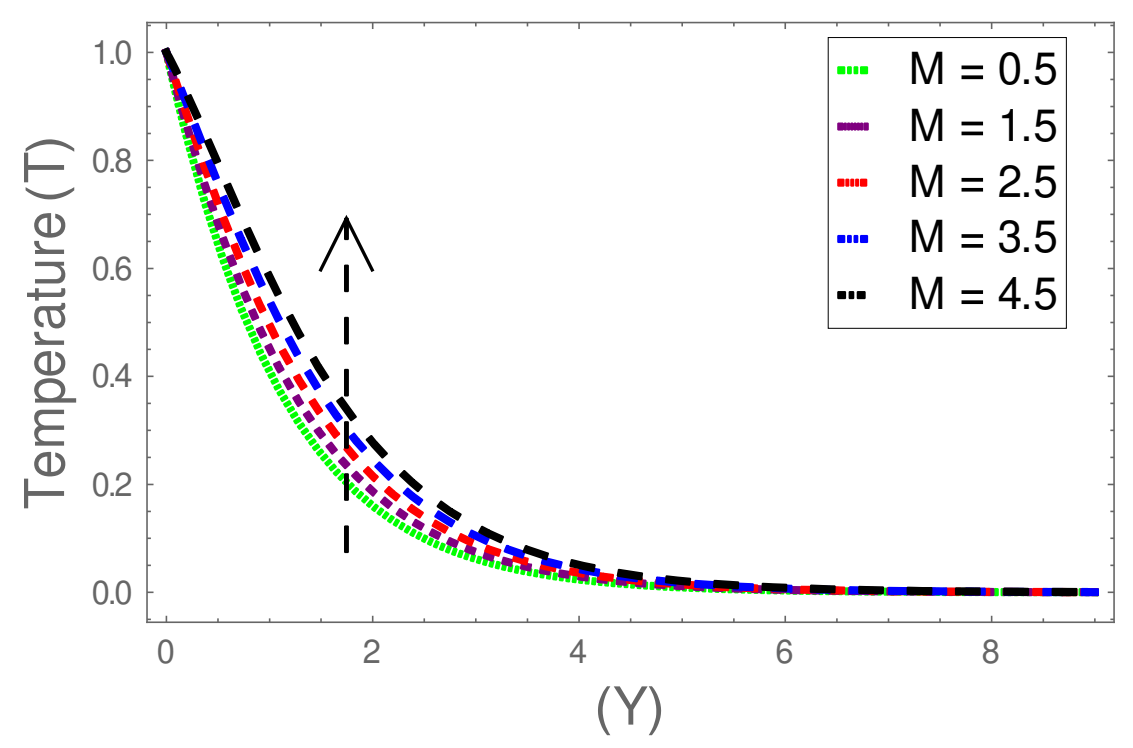

Fig. 6. Variation of $(M)$ on thermal field 


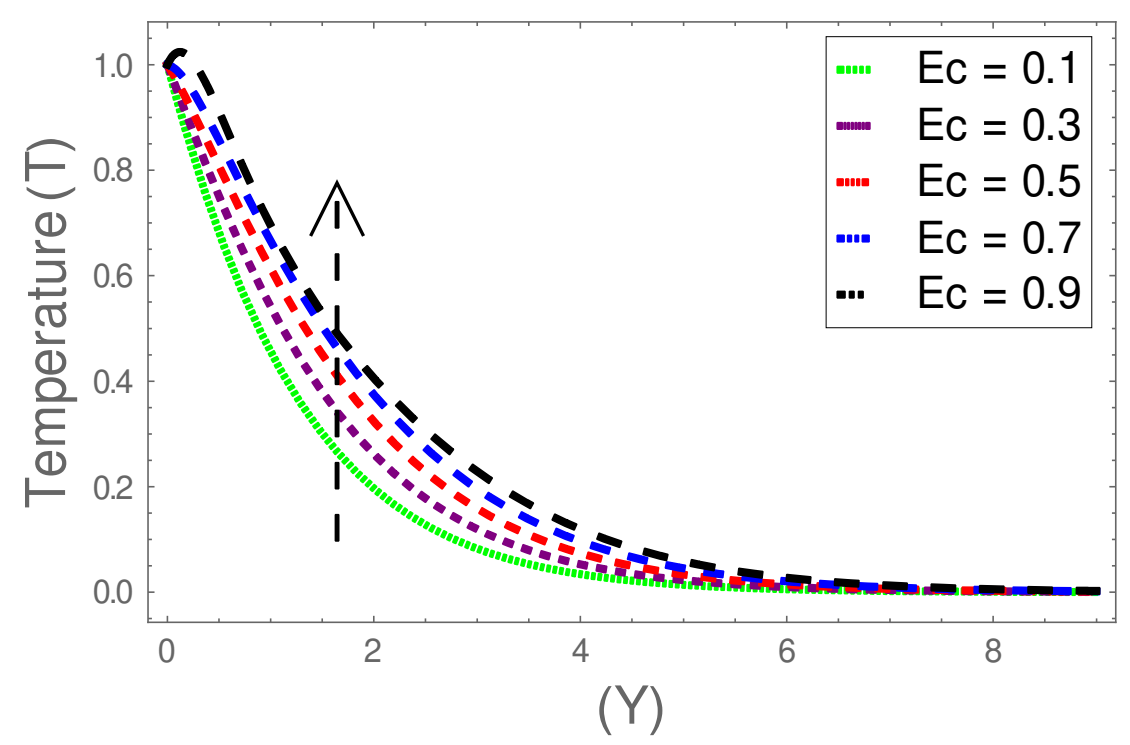

Fig. 7. Variation of $(\beta)$ on thermal field

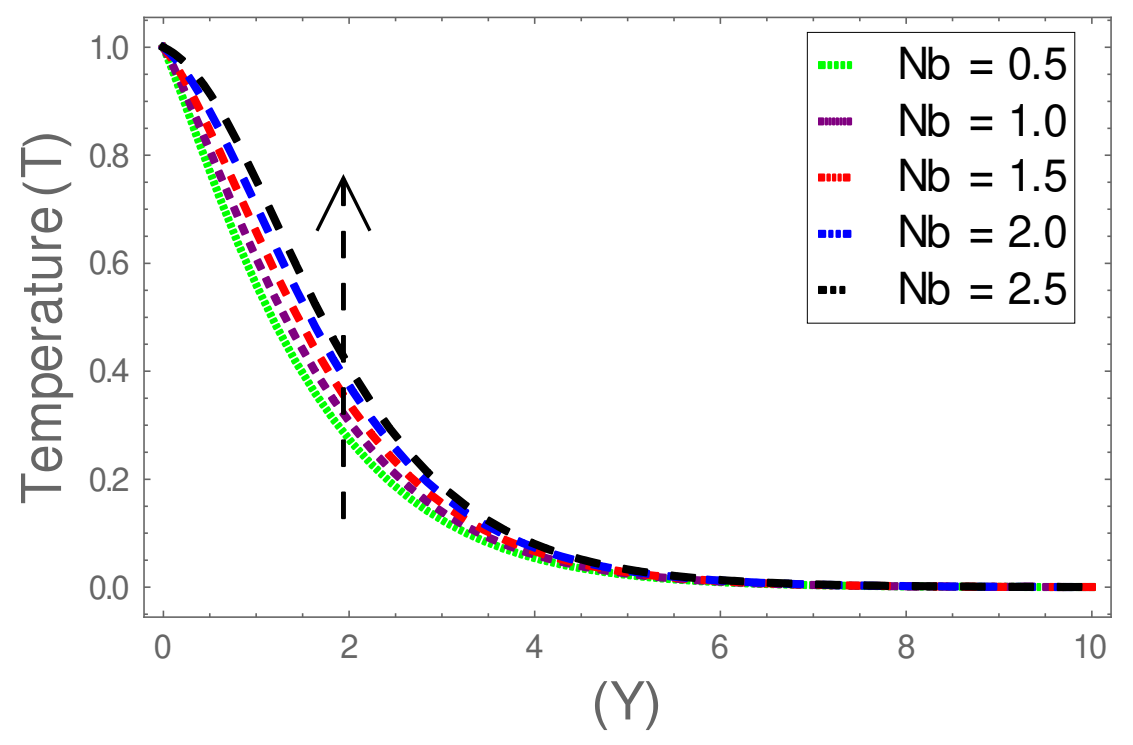

Fig. 8. Variation of $(\mathrm{Nb})$ on thermal field 


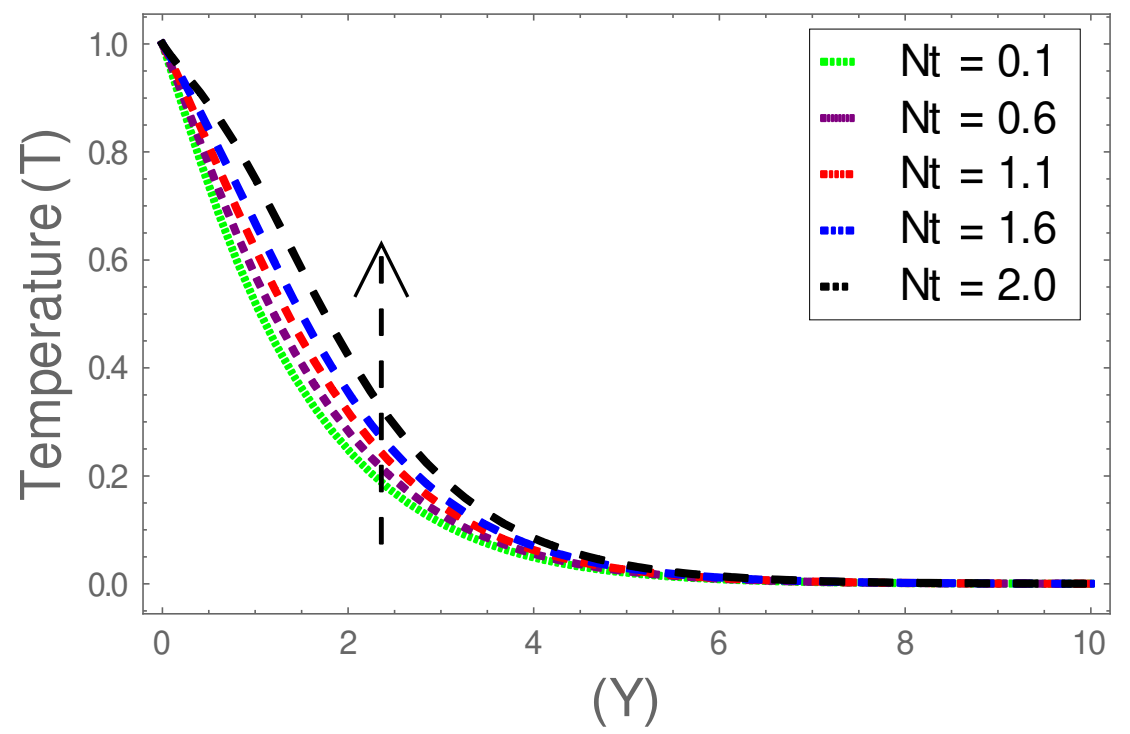

Fig. 9. Variation of $(N t)$ on thermal field

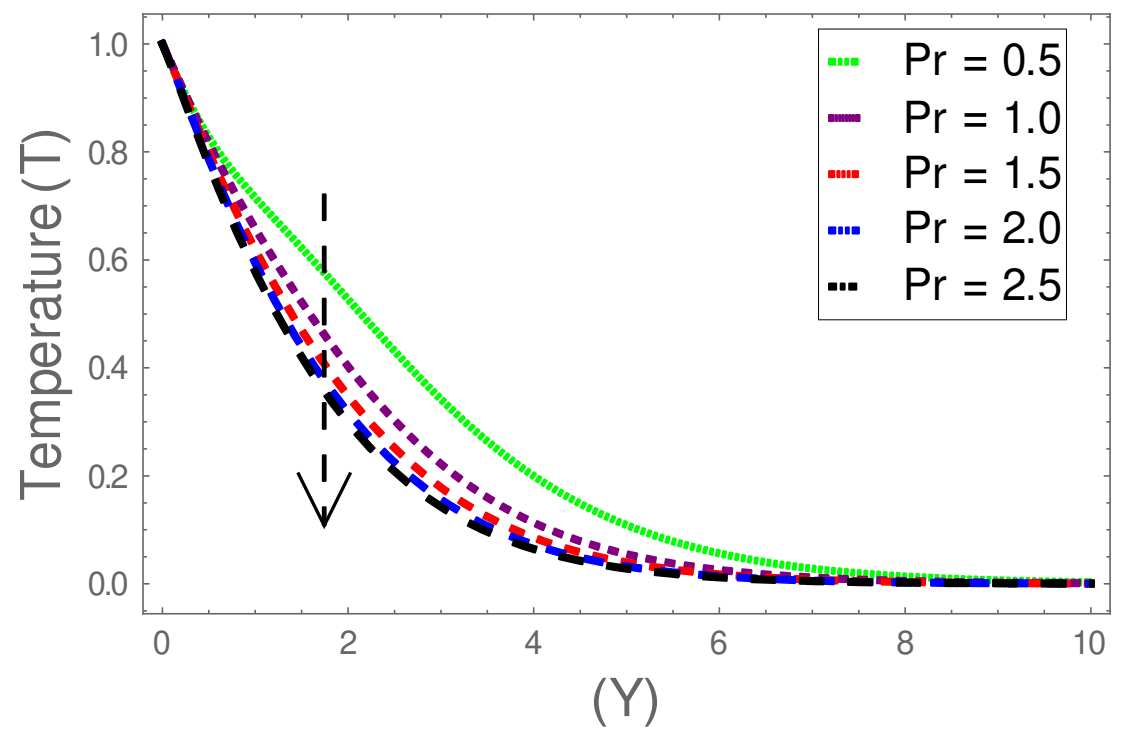

Fig. 10. Variation of $(\operatorname{Pr})$ on thermal field 


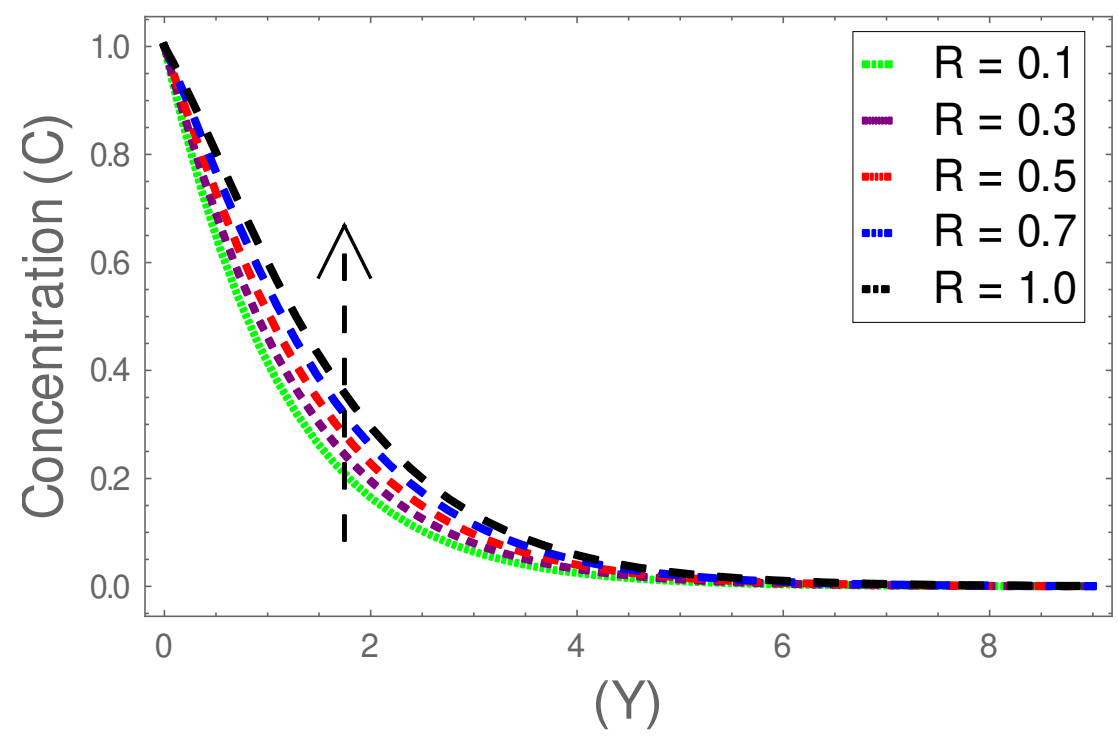

Fig. 11. Variation of $(R)$ on concentration profile

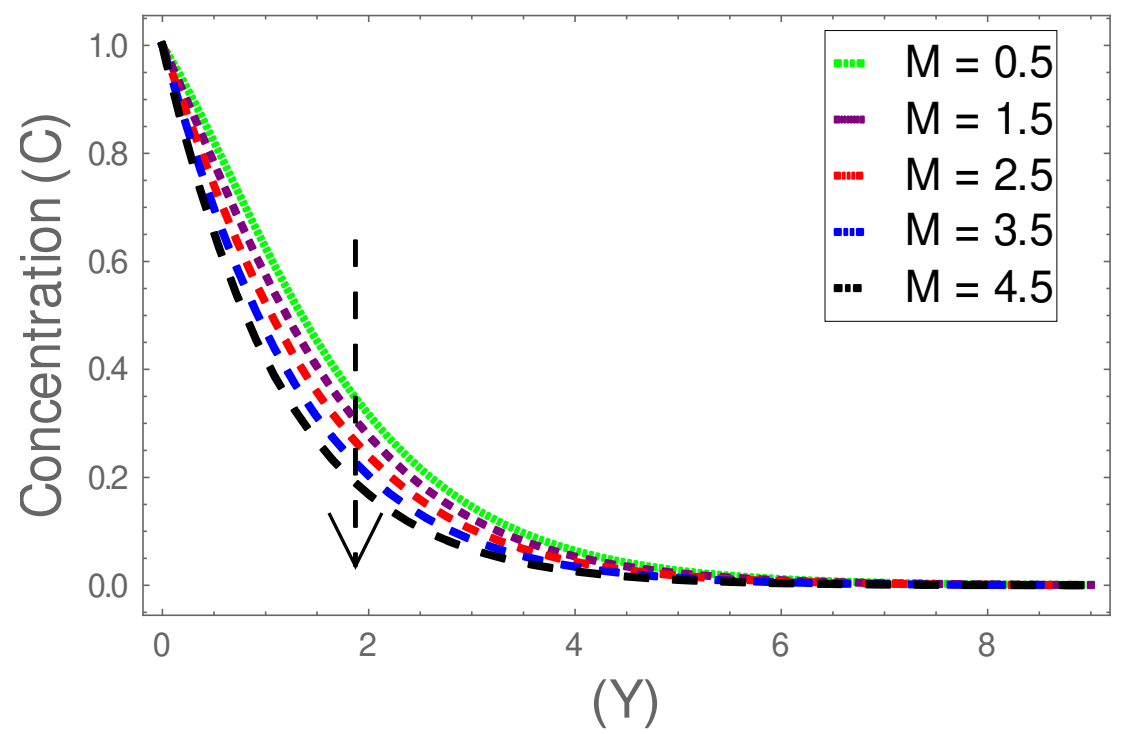

Fig. 12. Variation of $(M)$ on concentration profile 


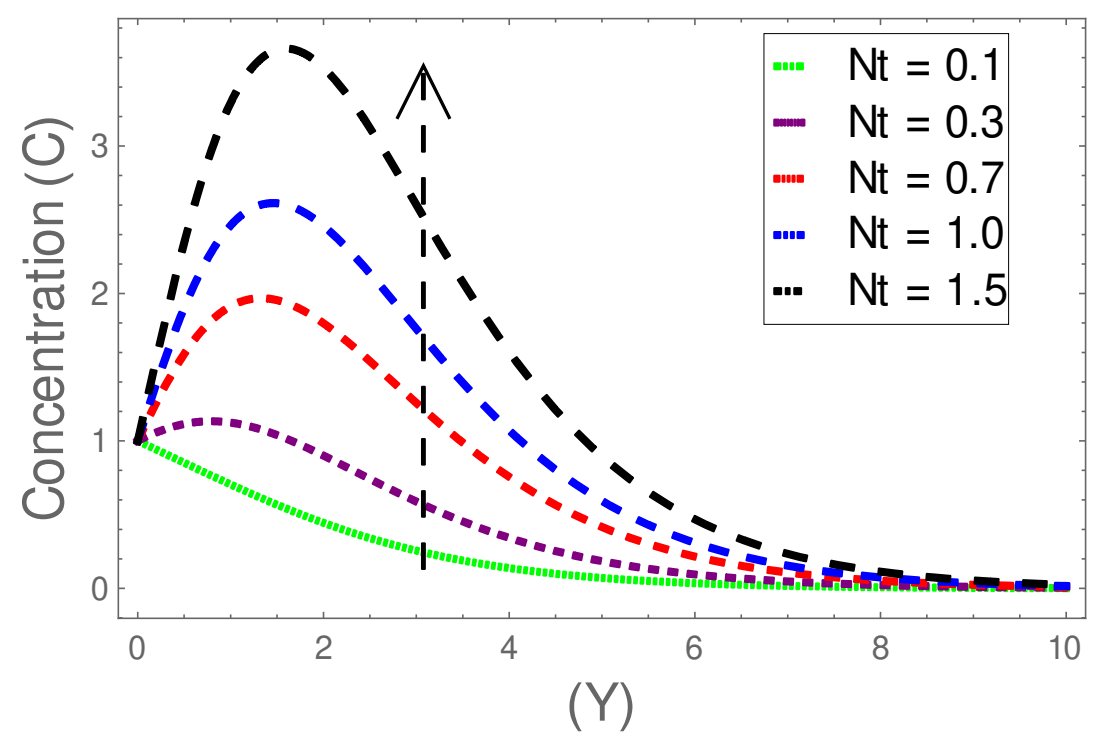

Fig. 13. Variation of $(\mathrm{Nt})$ on concentration profile

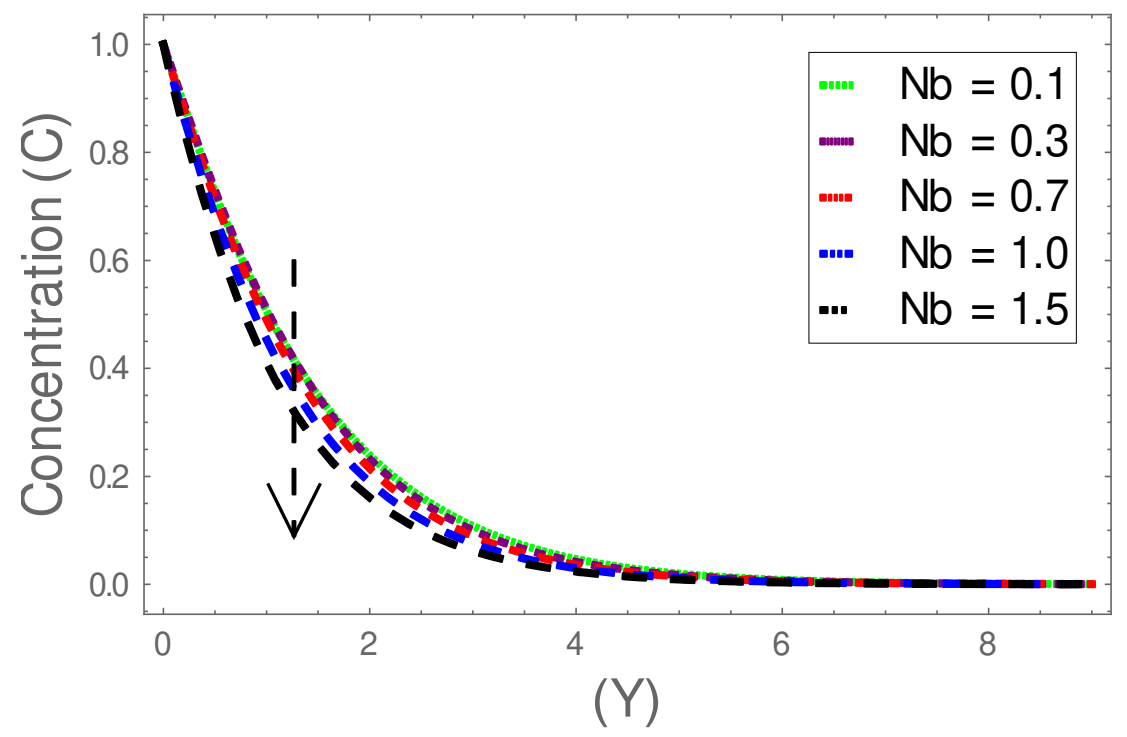

Fig. 14. Variation of $(\mathrm{Nb})$ on concentration profile 


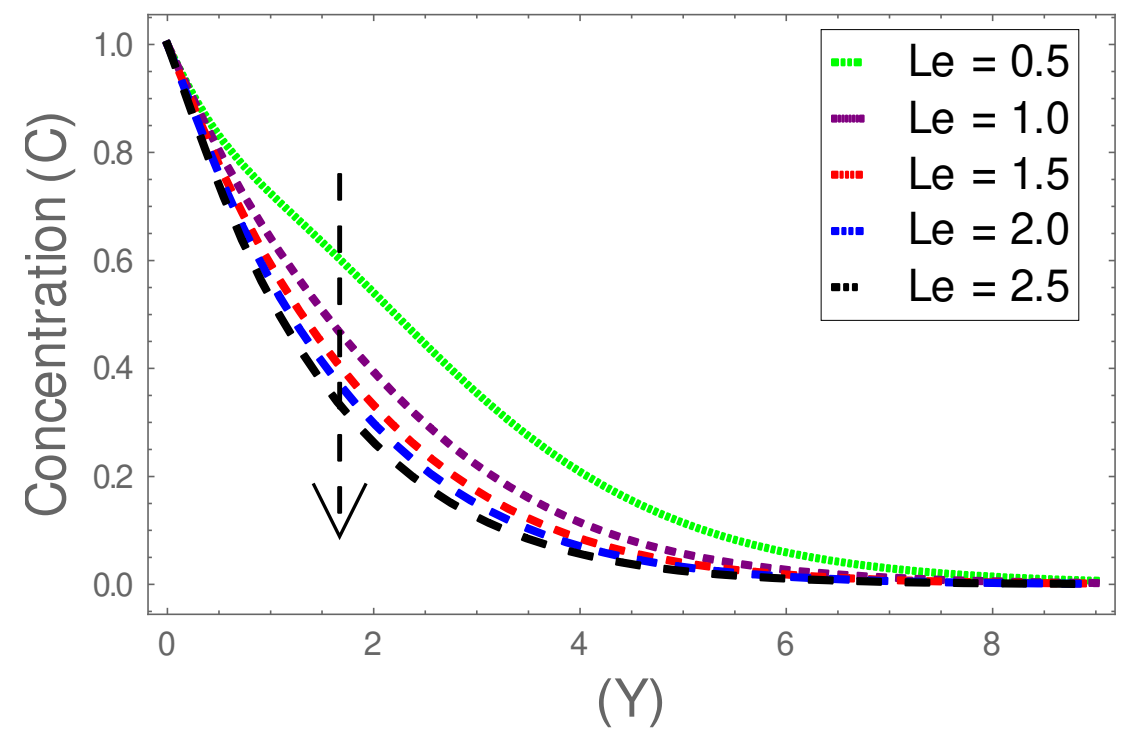

Fig. 15. Variation of $(L e)$ on concentration profile

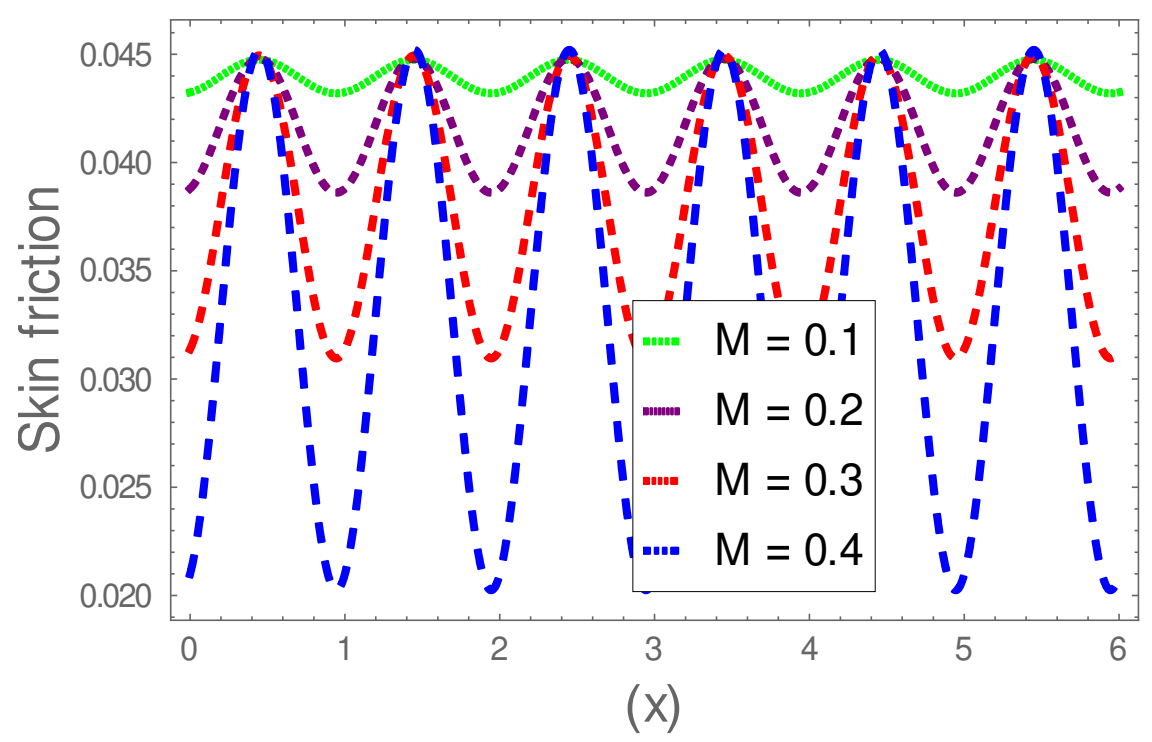

Fig.16 Skin friction illustration via $(M)$ 


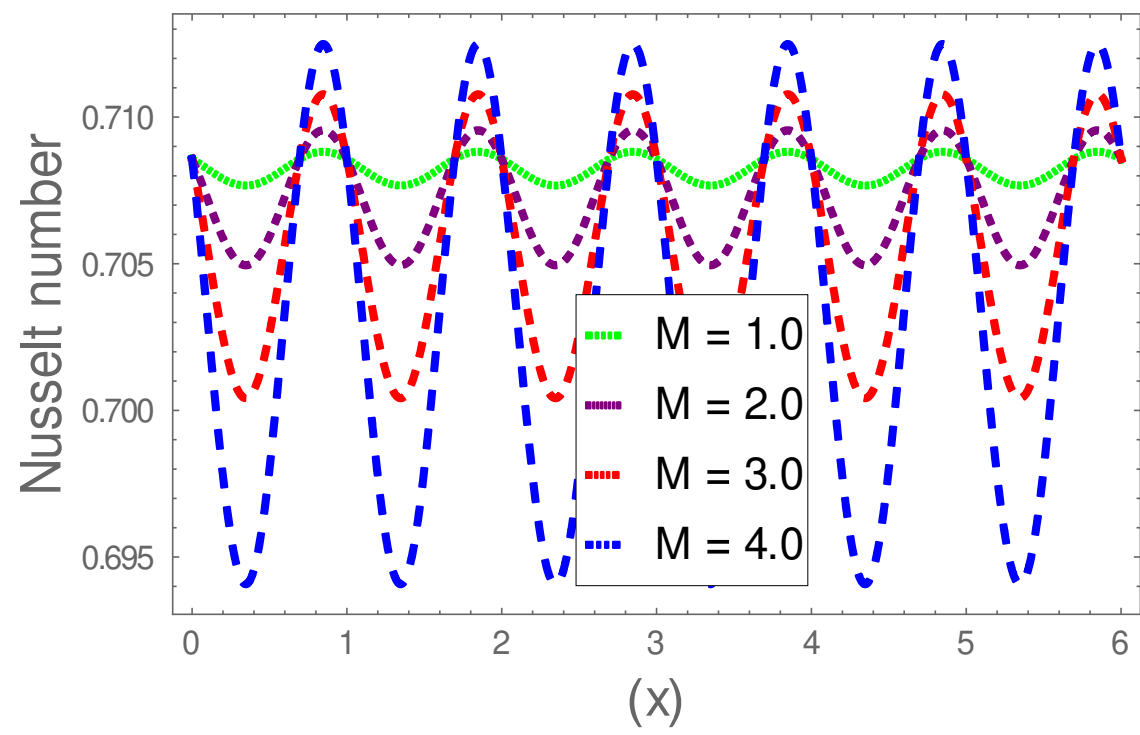

Fig.17 Nusselt number illustration via $(M)$

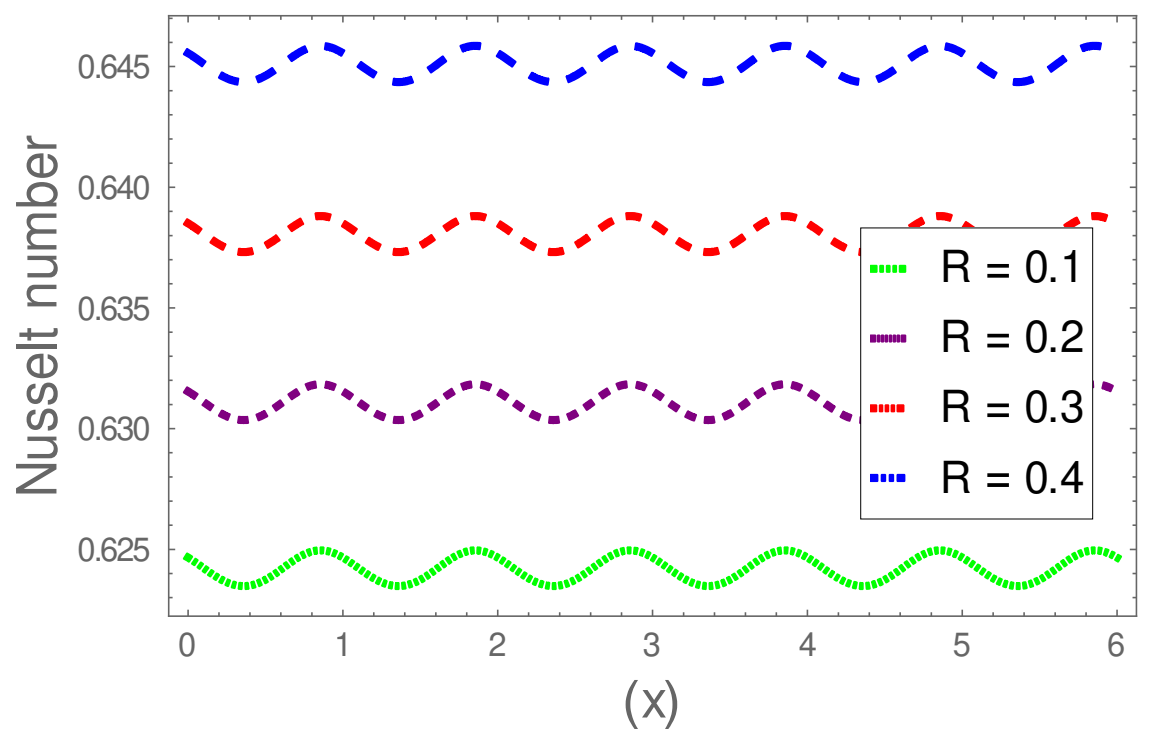

Fig.18 Nusselt number illustration via $(R)$ 


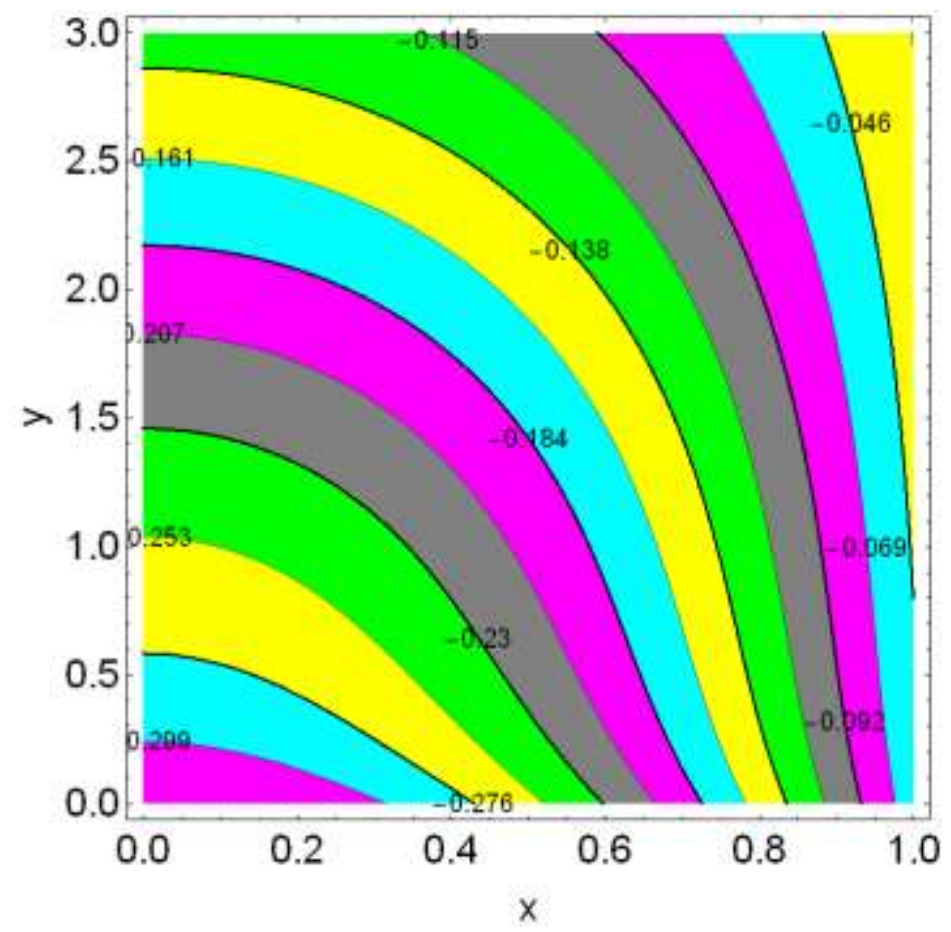

Fig. 19 Illustration of streamlines for $(M=1.0)$ and $(R=3.0)$

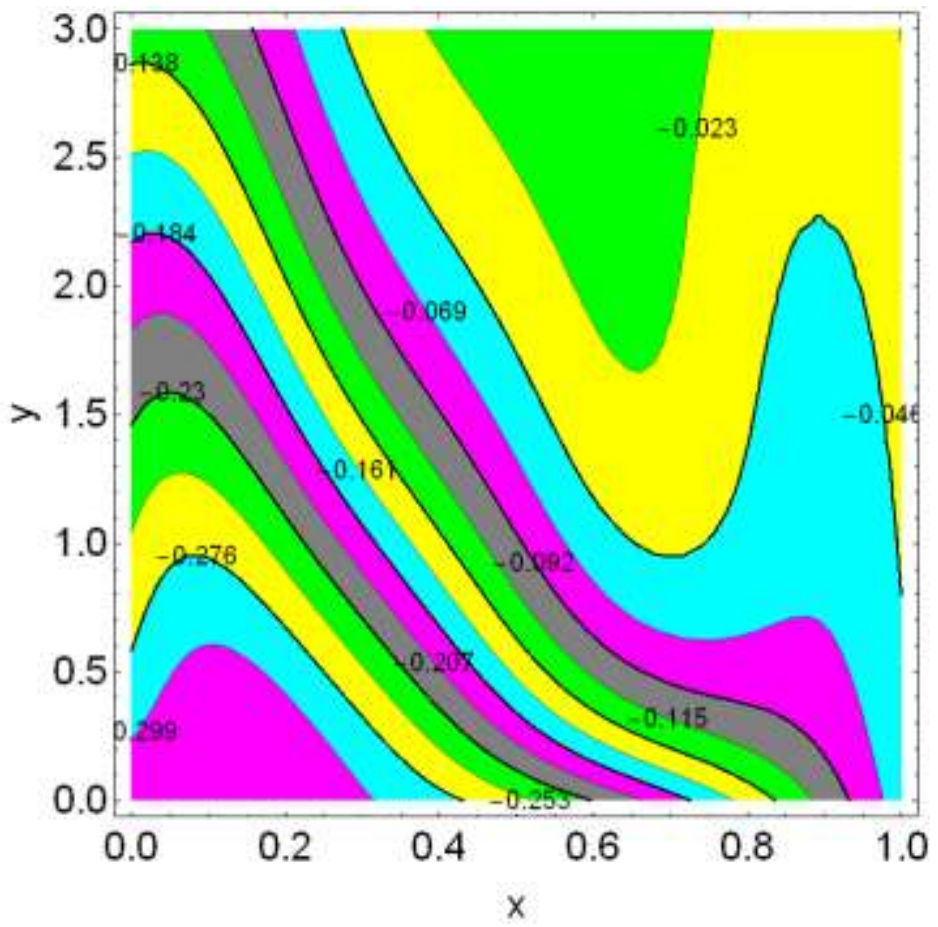

Fig. 20 Illustration of streamlines for $(M=4.0)$ and $(R=3.0)$ 


\section{Conclusion}

The present investigation displays the simulation of periodic magnetic Jeffrey nanofluid flow by a stretching surface. The fundamental flow laws diminished into dimensionless form via transformation and solved analytically with the implementation of homotopic method. One of the novel concerns is to perceive theoretically, how a periodic magnetic field affects nanoparticles. We witnessed following distinguished features through abovementioned investigation:

- It is observed that velocity field enhances subject to increment in thermal radiation constraint. On the other hand, velocity profile diminishes due to higher implication of magnetic field.

- Consideration of radiative factor and magnetic influence escalates thermal field curves.

- One can perceived that heat mass transference coefficient boosts through higher radiation constraint and diminishes with increment of magnetic parameter.

- An increment in Brownian motion parameter dwindles concentration profile and oppositive characteristics witnessed for thermophoretic force.

- Here, one can noticed that heat mass transference coefficient boosts through higher radiation constraint.

- The Nusselt number diminishes for higher values magnetic parameter and increases for larger radiation parameter.

- The uprising value of magnetic parameter reducing fluid velocity and heat transfer rate rapidly.

\section{Data availability statement}

The data used to support the findings of this study are included within the article.

\section{Conflict of interest}

The authors declared that they have no conflict of interest about this manuscript. 


\section{References}

1. Chen, H.; Ding, Y. Heat transfer and rheological behavior of nanofluids a review. Advances in Transport Phenomena, 2009, 1: 135-177.

2. Choi, S. U. S. Enhancing thermal conductivity of fluids with nanoparticles. The Proceedings of the 1995 ASME International Mechanical Engineering Congress and Exposition. San Francisce, USA, 1995, 99-105.

3. Rashidi, M. M.; Beg, O. A.; Asadi, M.; et al. DTM padé modeling of natural convective boundary layer flow of a nanofluid past a vertical surface. J Environ Eng (New York) 2012, 4, 13-24.

4. Turkyilmazoglu, M.; Pop, I. Heat and mass transfer of unsteady natural convection flow of some nanofluids past a vertical infinite flat plate with radiation effect. Int. J. Heat Mass Transf. 2013, 59, 167-171.

5. Khan, W. A.; Makinde; O. D. MHD nanofluid bioconvection due to gyrotactic microorganisms over a convectively heat stretching sheet. Int. J. Therm. Sci. 2014, 81, 118124.

6. Sheihkolelami, M.; Gorji, B. M.; Ganji, D. D. MHD free convection in an eccentric semi annulus filled with nanofluid. J Taiwan Inst Chem Eng 2014, 45, 1204-1216.

7. Sheihkolelami, M.; Gorji, B. M.; Ganji, D. D. Lattice Boltzmann method for MHD natural convection heat transfer using nanofluid. Powder Technol. 2014, 254, 82-93.

8. Imtiaz, M.; Hayat, T.; Hussain, M.; et al. Mixed convection flow of nanofluid with Newtonian heating. Eur. Phys. J. Plus 2014, 129, 1-11.

9. Hayat, T.; Abbasi, F. M.; Al-Yami, M.; et al. Slip and Joule heating effects in mixed convection peristaltic transport of nanofluid with Soret and Dufour effects. J. Mol. Liq. 2014, 194, 93-99.

10. Lin, Y.; Zheng, L.; Zheng, X. Radiation effects on Marangoni convection flow and heat transfer in pseudoplastic non-Newtonian nanofluids with variable thermal conductivity. Int. J. Heat Mass Transf. 2014, 77, 708-716.

11. Ahmed, A.; Poonam, K.K.; Khalil, M. et al. Numerical Scrutinization of Unsteady 3D Flow of Jeffrey Nanofluid with MHD in a Porous Medium. Int. J. Appl. Comput. Math 2021, 106 https://doi.org/10.1007/s40819-021-01031-4.

12. Fiza, M.; Alsubie, A.; Ullah, H.; Hamadneh, N.N.; Islam, S.; Khan, I. Three-dimensional rotating flow of MHD Jeffrey fluid flow between two parallel plates with impact of hall current. MATH PROBL ENG 2021, Article ID 6626411, https://doi.org/10.1155/2021/6626411.

13. Ahmad, I.; Aziz, S.; Ali, N.; Khan, S. Radiative unsteady hydromagnetic 3D flow model for Jeffrey nanofluid configured by an accelerated surface with chemical reaction. Heat Transfer 2021, 50, 942-965. DOI: 10.1002/htj.21912.

14. Rasheed, H.; AL-Zubaidi, A.; Islam, S.; Saleem, S.; Khan, Z.; Khan, W. Effects of Joule heating and viscous dissipation on magnetohydrodynamic boundary layer flow of Jeffrey nanofluid over a vertically stretching cylinder. Coatings 2021, 11, 353. https://doi.org/10.3390/coatings11030353.

15. Naidu, K.K.; Babu, D.H.; Reddy, S.H.; Narayana, P.V.S. Radiation and partial slip effects on magnetohydrodynamic Jeffrey nanofluid containing gyrotactic microorganisms over a 
stretching surface. J. Thermal Sci. Eng. Appl. 2021, $13 \quad 031011$ https://doi.org/10.1115/1.4048213.

16. Ge-JiLe, H.; Qayyum, S.; Shah, F.; Khan, M.I.; Khan, S. Slip flow of Jeffrey nanofluid with activation energy and entropy generation applications. Adv. Mech. Eng. 2021, 13, 19. DOI: $10.1177 / 16878140211006578$.

17. Noor, N.A.M.; Shafie, S.; Admon, M.A. Heat and mass transfer on MHD squeezing flow of Jeffrey nanofluid in horizontal channel through permeable medium. PLoS One 2021, https://doi.org/10.1371/journal.pone.0250402.

18. Shahzada, F.; Sagheer, M.; Hussain, S. Numerical simulation of magnetohydrodynamic Jeffrey nanofluid flow and heat transfer over a stretching sheet considering Joule heating and viscous dissipation. AIP Adv. 2018, 8, 065316 https://doi.org/10.1063/1.5031447.

19. Hayat, T.; Khan, M.I.; Qayyum, S.; et al. Entropy generation in flow with silver and copper nanoparticles. Colloid Surf A 2018, 539, 335-346.

20. Krishnamurthy, M.R.; Prasannakumara, B.C.; Gorla; R.S.R.; et al. Non-linear thermal radiation and slip effect on boundary layer flow and heat transfer of suspended nanoparticles over a stretching sheet embedded in porous medium with convective boundary conditions. J Nanofluids 2016, 5, 522-530.

21. Kumar, K.G.; Gireesha, B.J.; Kumara, B.C.; et al. Impact of chemical reaction on marangoni boundary layer flow of a Casson nano liquid in the presence of uniform heat source sink. Diffus Found 2017, 11, 22-32.

22. Roja, A.; Gireesha, B.J.; Prasannakumara, B.C. MHD micropolar nanofluid flow through an inclined channel with entropy generation subjected to radiative heat flux, viscous dissipation and multiple slip effects. Multidiscip Model Mater Struct 2020, 16, 1475-1496.

23. Hamid, M.; Khan, Z.H. Khan, W.A.; et al. Natural convection of water-based carbon nanotubes in partially heated rectangular fin-shaped cavity with inner cylindrical obstacle. Phys Fluids 2019, 31, 103607.

24. Khan, Z.H.; Usman, M.; Zubair, T.; et al. Brownian motion and thermophoresis effects on unsteady stagnation point flow of Eyring-Powell nanofluid: a Galerkin approach. Commun Theor Phys 2020, 72, 125005.

25. Khan, Z.H.; Khan, W.A.; Sheremet, M.A. Enhancement of heat and mass transfer rates through various porous cavities for triple convective-diffusive free convection. Energy 2020, 201, 117702.

26. Riaz, A.; Zeeshan, A.; Bhati, M.M.; Ellahi, R. Peristaltic propulsion of Jeffrey nano-liquid and heat transfer through a symmetrical duct with moving walls in a porous medium. Physica A: Statistical Mechanics and its Applications, vol. 545, 2020.

27. Riaz, A.; Ellahi, R.; Sait, S.M.; Muhammad, T. Magnetized Jeffrey nanofluid with energy loss in between an annular part of two micro non concentric pipes. Energy Sources, 2020.

28. Fiza, M.; Ullah, H.; Islam, S. Three-dimensional MHD rotating flow of viscoelastic nanofluid in porous medium between parallel plates. Journal of Porous Media 2020, 23, 715-729.

29. Rasheed, H.; Islam, S.; Khan, Z.; Alharbi, S.O.; Khan, W.; Alotaibi, H.; Khan, I. Numerical simulation of electrically conducting and thermally radiative nanofluid flow in view of elongated slippery plates. AIP Adv. 2021, 11, 065019. https://doi.org/10.1063/5.0046636. 
30. Islam, S.; Rasheed, H.; Nisar, K.S.; Alshehri, N.A.; Zakarya, M. Numerical simulation of heat mass transfer effects on MHD flow of Williamson nanofluid by a stretching surface with thermal conductivity and variable thickness. Coatings 2021, 11, 684; https://doi.org/10.3390/coatings11060684.

31. Rasheed, H.; Islam, S.; Khan, Z.; Alharbi, S.O.; Alotaibi, H.; Khan, I. Complexity 2021, 2021, Article ID 9951162, 9 pages. https://doi.org/10.1155/2021/9951162

\section{Authors Contribution Statement}

H.R and Z.K wrote main manuscript file S.I and W.K give simulation of the problem. All authors contributed equally. 